\title{
Sleep deficiency and motor vehicle crash risk in the general population: $a$ prospective cohort study
}

\author{
Daniel J. Gottlieb ${ }^{1,2,3^{*}}$, Jeffrey M. Ellenbogen ${ }^{3,4}$, Matt T. Bianchi ${ }^{3,4}$ and Charles A. Czeisler ${ }^{1,3}$
}

\begin{abstract}
Background: Insufficient sleep duration and obstructive sleep apnea, two common causes of sleep deficiency in adults, can result in excessive sleepiness, a well-recognized cause of motor vehicle crashes, although their contribution to crash risk in the general population remains uncertain. The objective of this study was to evaluate the relation of sleep apnea, sleep duration, and excessive sleepiness to crash risk in a community-dwelling population.

Methods: This was a prospective observational cohort study nested within the Sleep Heart Health Study, a communitybased study of the health consequences of sleep apnea. The participants were 1745 men and 1456 women aged 40-89 years. Sleep apnea was measured by home polysomnography and questionnaires were used to assess usual sleep duration and daytime sleepiness. A follow-up questionnaire 2 years after baseline ascertained driving habits and motor vehicle crash history. Logistic regression analysis was used to examine the relation of sleep apnea and sleep duration at baseline to the occurrence of motor vehicle crashes during the year preceding the follow-up visit, adjusting for relevant covariates. The population-attributable fraction of motor vehicle crashes was estimated from the sample proportion of motor vehicle crashes and the adjusted odds ratios for motor vehicle crash within each exposure category.
\end{abstract}

Results: Among 3201 evaluable participants, 222 (6.9\%) reported at least one motor vehicle crash during the prior year. A higher apnea-hypopnea index $(p<0.01)$, fewer hours of sleep $(p=0.04)$, and self-reported excessive sleepiness $(p<0.01)$ were each significantly associated with crash risk. Severe sleep apnea was associated with a $123 \%$ increased crash risk, compared to no sleep apnea. Sleeping 6 hours per night was associated with a 33\% increased crash risk, compared to sleeping 7 or 8 hours per night. These associations were present even in those who did not report excessive sleepiness. The population-attributable fraction of motor vehicle crashes was 10\% due to sleep apnea and 9\% due to sleep duration less than 7 hours.

Conclusions: Sleep deficiency due to either sleep apnea or insufficient sleep duration is strongly associated with motor vehicle crashes in the general population, independent of self-reported excessive sleepiness.

Keywords: Motor vehicle crash, Motor vehicle accident, Sleep deficiency, Sleep apnea, Sleep duration, Sleep deprivation, Epidemiology

\footnotetext{
* Correspondence: djgottlieb@partners.org

${ }^{1}$ Division of Sleep and Circadian Disorders, Departments of Medicine and

Neurology, Brigham \& Women's Hospital, 221 Longwood Ave, BLI 225E,

Boston, MA 02115, USA

${ }^{2}$ VA Boston Healthcare System, Boston, MA, USA

Full list of author information is available at the end of the article
}

(c) The Author(s). 2018 Open Access This article is distributed under the terms of the Creative Commons Attribution 4.0 International License (http://creativecommons.org/licenses/by/4.0/), which permits unrestricted use, distribution, and reproduction in any medium, provided you give appropriate credit to the original author(s) and the source, provide a link to the Creative Commons license, and indicate if changes were made. The Creative Commons Public Domain Dedication waiver (http://creativecommons.org/publicdomain/zero/1.0/) applies to the data made available in this article, unless otherwise stated. 


\section{Background}

Sleep deficiency, defined as insufficient "quantity or quality of sleep" for optimal health and performance, may result from inadequate total sleep duration or from fragmentation of sleep [1]. The potential health impact of insufficient sleep duration was first reported in the 1960s, when a general population survey of over one million adults found that individuals who reported sleeping fewer than 7 hours per night had increased mortality compared to those sleeping 7 to 8 hours per night $[2,3]$. Recent data indicate that usual sleep duration has decreased substantially since that time, with an estimated $25-30 \%$ of U.S. adults now sleeping 6 or fewer hours per night, a sleep duration associated with obesity, hypertension, glucose intolerance, diabetes mellitus, coronary heart disease, and death [4]. Restricted sleep is also an important cause of excessive sleepiness, a wellrecognized cause of motor vehicle crashes [5-7]. Data from the U.S. Centers for Disease Control indicate that habitual sleep duration of 6 or fewer hours per night is associated with a 2.6-fold higher risk of reporting having fallen asleep while driving, compared to those sleeping 7-9 hours per night, although motor vehicle crashes were not assessed [8]. Although there are individual differences in impairment from sleep deprivation [9], the perception of impairment quickly plateaus in individuals whose sleep duration is chronically restricted, despite continued declines in objective measures of performance $[10,11]$, suggesting that individuals may be unaware of their degree of impairment from sleep deficiency.

Obstructive sleep apnea is a common chronic disorder in adults and it is associated with excessive sleepiness [12]. The prevalence of sleep apnea has increased with the increase in overweight and obesity. Estimates based on current U.S. population demographics indicate that $17 \%$ of women and $34 \%$ of men aged $30-70$ have sleep apnea [13], with 5\% and 14\%, respectively, having both sleep apnea and self-reported excessive sleepiness. Like insufficient sleep duration, sleep apnea is associated with hypertension, diabetes mellitus, cardiovascular disease, and mortality [4, 14-17]. Sleep apnea is also associated with motor vehicle crash risk [18]. A recent metaanalysis concluded that patients with obstructive sleep apnea have a crash risk approximately 2.5 times that of individuals without sleep apnea, but the data were equivocal whether the excess crash risk was explained solely on the basis of excessive sleepiness [19]. While a diagnosis of obstructive sleep apnea can be made in the absence of excessive sleepiness [20], the importance of asymptomatic sleep apnea has been questioned. A recent U.S. Preventive Services Task Force recommendation concluded that "the current evidence is insufficient to assess the balance of benefits and harms of screening for obstructive sleep apnea in asymptomatic adults" [21].
As self-perceived excessive sleepiness is absent in more than half of individuals with sleep apnea in the general population, even among those with severe sleep apnea $[13,22]$, it remains an important unresolved question whether these individuals are at increased motor vehicle crash risk. Similarly, it has been argued that some individuals are naturally short sleepers and that failure to obtain an optimal duration of sleep is inconsequential in the absence of subjective sleepiness. In the present analysis, we ask whether individuals with sleep deficiency, due either to short usual sleep duration or obstructive sleep apnea, are at increased risk of motor vehicle crashes, independent of self-reported daytime sleepiness.

\section{Methods}

\section{Study design}

The Sleep Heart Health Study is a community-based prospective cohort study of the cardiovascular consequences of sleep apnea. Briefly, 6441 men and women aged 40 years and older were recruited from existing populationbased studies, as previously described [23, 24]. Therapy for sleep apnea or use of nocturnal supplemental oxygen were exclusion criteria. At baseline, participants completed sleep habits and general health questionnaires and underwent overnight polysomnography. Approximately 2 years later, the participants again completed sleep habits and general health questionnaires, including questions related to driving habits and history of motor vehicle crashes. The aim of the present investigation is to determine whether sleep apnea or short sleep duration are associated with increased crash risk in the general population, and whether self-reported sleepiness identifies those at increased crash risk. The protocol was approved by the Institutional Review Board of each participating center and signed informed consent was provided by each participant. The full Sleep Heart Health Study protocol, including the sleep habits, sleepiness and driving history questionnaires, are available at http://jhuccs1.us/shhs/details/studydoc.htm.

\section{Study sample}

The composition of the study sample is shown in Fig. 1. Of the 6441 Sleep Heart Health Study participants, 2-year follow-up questionnaire data were available for 5494 (85.3\%). Of these, 624 non-drivers were excluded, as were 238 with missing data on sleepiness, sleep duration, or motor vehicle accidents. Of the remaining 4632 individuals, 3201 reported the average number of miles driven per year. Given the strong association between miles driven and motor vehicle crash risk, these 3201 individuals are the primary sample analyzed for this report. The included sample was slightly younger (62.3 vs. 63.5 years, $p<0.001$ ) and had a higher percentage of men $(54.5 \%$ vs. $39.9 \%, p<0.001)$ than the 3240 


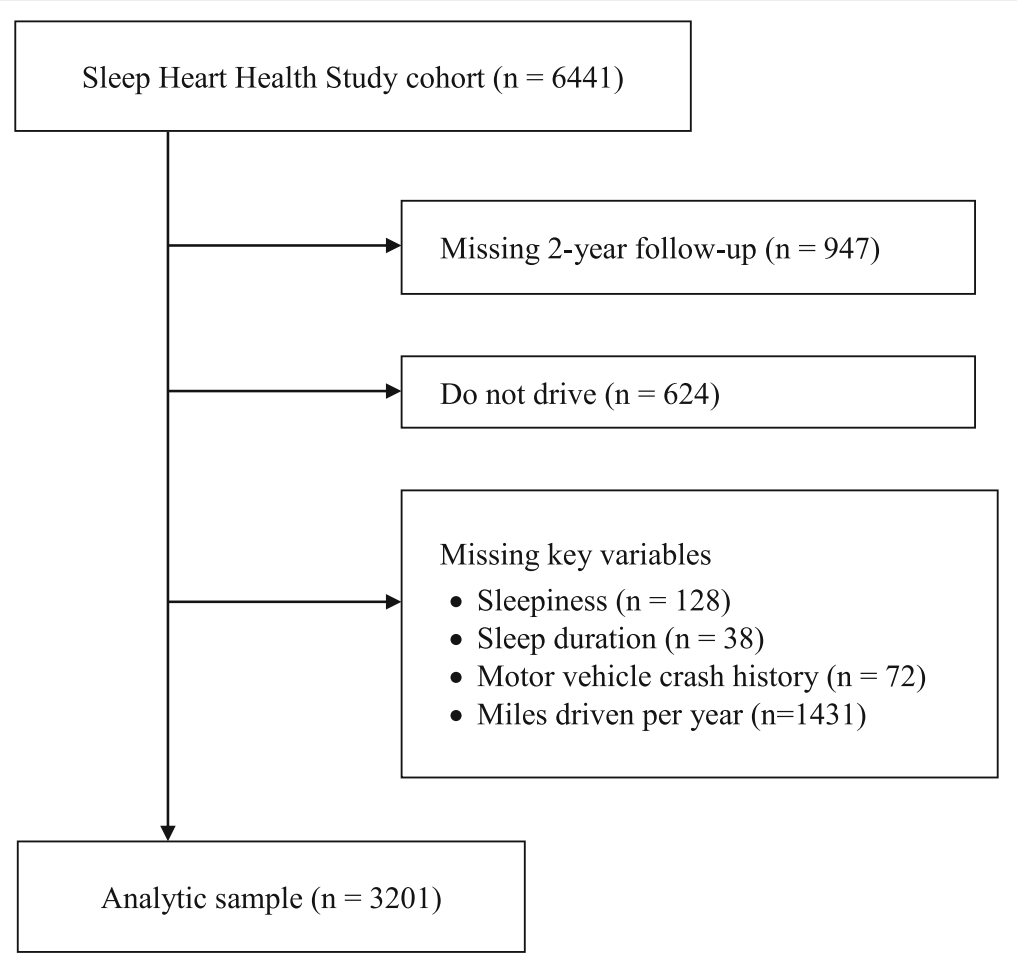

Fig. 1 Ascertainment of the study sample

participants who were excluded for any reason. Their reported usual sleep duration was slightly longer (7.1 vs. 7.0 hours/night, $p<0.01$ ), and they were similar to excluded participants in mean apnea-hypopnea index (AHI, 8.7 vs. 9.0, $p=0.36$ ) and Epworth Sleepiness Scale scores (7.9 vs 7.7, $p=0.34$ ). Of the 3201 included participants, 2340 reported the usual number of hours driven per day, while 1295 participants were excluded because they did not report the number of miles driven per year but did report the usual number of hours driven per day. There was no significant difference between included and excluded participants in reported usual hours of driving per day (1.83 versus 1.86 hours per day, $p=0.54$ ).

\section{Sleep measures}

Participants underwent a single night of in-home polysomnography at baseline, as previously described $[25,26]$. AHI was defined as the average number of apneas plus hypopneas per hour of sleep, where both apneas and hypopneas required an associated $4 \%$ or greater oxygen desaturation. Usual sleep duration was defined as the response to the question, "How many hours of sleep do you usually get at night (or your main sleep period) on weekdays or workdays?" Responses were integer values. The stability of this measure over time in this cohort has been previously reported [27]. Sleepiness was assessed using the Epworth Sleepiness Scale, an eight-item self-completion questionnaire that asks the participant to rate his/her likelihood of falling asleep in a variety of commonly encountered situations [28]. Excessive sleepiness was defined as a score $\geq 11$ on this scale.

\section{Driving history}

As part of a general follow-up questionnaire completed approximately 2 years after baseline, participants were asked, "Do you drive?" Those answering affirmatively were further asked about driving frequency, average time spent driving per day or miles driven per year, and the question: "How many accidents have you had in the last year while you were the driver?"

\section{Statistical analysis}

All statistical analyses were performed using SAS version 9.2 (SAS Institute, Inc., Cary, NC). Descriptive statistics are presented comparing participants with and without motor vehicle crash, with independent sample $t$ tests used to compare means of continuous variables and Fisher's exact test to compare proportions. Logistic regression analysis was used to examine the relation of sleep apnea and sleep duration at baseline to the occurrence of at least one motor vehicle crash during the year preceding the follow-up visit, adjusting for relevant covariates. The primary models adjusted for age, sex, and miles driven. The Epworth Sleepiness Scale score was added to the models to assess mediation by self- 
reported sleepiness of the effects of sleep apnea and usual sleep duration, and models restricted to those without excessive sleepiness were run to determine whether excess crash risk was present in those without self-reported sleepiness. Models that included both sleep apnea and sleep duration measures, with an appropriate interaction term, were used to assess a possible synergy between sleep apnea and sleep deprivation in relation to crash risk. Sensitivity analyses including those individuals missing data on miles driven, adjusted only for age and sex, were conducted to determine whether their exclusion might have biased the study results. Additional models adjusted for alcohol consumption, smoking history, body mass index, sleeping pill use, and caffeine consumption; explored the independent effect of habitual snoring on crash risk; explored possible sex differences in the effect of sleep deficiency on crash risk; and explored differences in the effect of sleep deficiency between older and younger participants. The populationattributable fraction of motor vehicle crashes was estimated from the sample proportion of motor vehicle crashes in each exposure category and the adjusted odds ratios for motor vehicle crash in those categories, as described by Miettinen [29].

\section{Results}

At least one motor vehicle crash during the year prior to follow-up evaluation was reported by $6.9 \%$ of the 3201 participants. Motor vehicle crashes were somewhat more common in men than in women and were significantly associated with number of miles driven per year, AHI, sleep duration, and self-reported sleepiness (Table 1). Adjusted for age, sex, and miles driven, the odds ratio for any motor vehicle crash increased by $15 \%$ for every 10-unit increase in AHI in the overall population and by $17 \%$ for every 10-unit increase in AHI in participants who did not report excessive sleepiness (Table 2). Although non-linear models were not a significantly better fit to the data, the excess risk in self-reported non-sleepy participants was apparent only in those with severe sleep apnea (Fig. 2a). Compared to those without sleep apnea (AHI $<5$, $n=1730$ ), those with mild (AHI 5 to $<15, n=913$ ), moderate (AHI 15 to $<30, n=364$ ), and severe (AHI $\geq 30$, $n=194)$ sleep apnea had adjusted odds ratios (aOR) for any motor vehicle crash that were respectively $7 \%, 13 \%$ and $123 \%$ higher. The population-attributable fraction of motor vehicle crashes due to sleep apnea was $10 \%$. Habitual snoring was not significantly associated with crash risk in models that included measures of sleep apnea.

Table 1 Baseline characteristics of the study sample

\begin{tabular}{|c|c|c|c|}
\hline Characteristic & No MVC $(n=2979)^{*}$ & $\operatorname{MVC}(n=222)^{\dagger}$ & $P$ value \\
\hline Age - years & $62.3(10.1)$ & $61.5(10.8)$ & 0.22 \\
\hline Male sex - no. (\%) & $1610(54.0)$ & $135(60.8)$ & 0.06 \\
\hline Body mass index $-\mathrm{kg} / \mathrm{m}^{2}$ & $28.2(4.9)$ & $28.6(5.6)$ & 0.31 \\
\hline \multicolumn{4}{|l|}{ Comorbid illness - no. (\%) } \\
\hline Diabetes mellitus & $192(6.7)$ & $15(7.3)$ & 0.66 \\
\hline Hypertension & $1174(39.4)$ & $83(37.4)$ & 0.57 \\
\hline Myocardial infarction & $186(6.3)$ & $11(5.0)$ & 0.34 \\
\hline Stroke & $73(2.5)$ & $5(2.3)$ & 0.65 \\
\hline Smoking status - no. (\%) & & & 0.96 \\
\hline Current & $297(10.0)$ & $21(9.5)$ & \\
\hline Former & $1367(46.1)$ & $103(46.4)$ & \\
\hline Alcohol consumption - drinks per week & $1(0,4)$ & $0(0,3)$ & 0.88 \\
\hline Caffeine consumption - drinks per day & $2(1,4)$ & $2(1,4)$ & 0.76 \\
\hline Sleeping pill use - no. (\%) & $1989(6.7)$ & $19(8.6)$ & 0.27 \\
\hline Epworth Sleepiness Scale score & $7.7(4.3)$ & $8.6(4.7)$ & $<0.01$ \\
\hline Apnea-hypopnea index - events per hour & $4.3(1.3,10.9)$ & $5.5(1.3,13.7)$ & $<0.01$ \\
\hline Usual sleep duration - hours per night & $7.1(1.1)$ & $6.9(1.1)$ & 0.02 \\
\hline Miles driven per year - thousands & $9.0(4.0,14.0)$ & $11.5(7.0,15.0)$ & $<0.001$ \\
\hline
\end{tabular}

Values are number (\%) or mean (standard deviation), with the exception of alcohol and caffeine consumption, apnea-hypopnea index, and miles driven, which are median (first quartile, third quartile)

MVC motor vehicle crash

"Missing data in the sample without MVC: body mass index $(n=4)$, diabetes $(n=91)$, myocardial infarction $(n=26)$, stroke $(n=26)$, smoking status $(n=16)$, alcohol consumption ( $n=206)$, sleeping pill use $(n=6)$

${ }^{\dagger}$ Missing data in the sample with MVC: body mass index $(n=3)$, diabetes $(n=16)$, myocardial infarction $(n=1)$, stroke $(n=1)$, alcohol consumption ( $\left.n=24\right)$, sleeping pill use $(n=1)$ 
Table 2 Relation of sleep apnea, sleep duration, and self-reported sleepiness to motor vehicle crash risk

\begin{tabular}{|c|c|c|}
\hline Characteristic & $\begin{array}{l}\text { All participants } \\
(n=3201)\end{array}$ & $\begin{array}{l}\text { Participants without } \\
\text { excessive sleepiness } \\
(n=2402)\end{array}$ \\
\hline \multirow{2}{*}{$\begin{array}{l}\text { Apnea-hypopnea index } \\
\text { - per } 10 \text { events/hour }\end{array}$} & $1.15(1.04,1.26)$ & 1.17 (1.02 1.33) \\
\hline & $P<0.01$ & $P=0.02$ \\
\hline \multirow{2}{*}{$\begin{array}{l}\text { Usual sleep duration } \\
\text { - per hour less sleep }\end{array}$} & $1.13(1.01,1.28)$ & $1.22(1.05,1.43)$ \\
\hline & $P=0.04$ & $P<0.01$ \\
\hline \multirow{2}{*}{$\begin{array}{l}\text { Excessive daytime } \\
\text { sleepiness (Epworth } \\
\text { Sleepiness Scale } \\
\text { score } \geq 11 \text { ) }\end{array}$} & $1.54(1.15,2.07)$ & - \\
\hline & $P<0.01$ & \\
\hline
\end{tabular}

Values are odds ratio ( $95 \%$ confidence interval), adjusted for age, sex, and miles driven per year

"Absence of excessive sleepiness defined as Epworth Sleepiness Scale Score $\leq 10$
For every hour decrease in usual sleep duration, the aOR for any motor vehicle crash increased by $13 \%$ in the overall population and by $22 \%$ in participants who did not report excessive sleepiness (Table 2). Crash risk was similar in those who reported sleeping 7 or 8 hours per night. Compared to those sleeping 7 or 8 hours per night $(n=2105)$, those who reported sleeping only 6 hours per night $(n=626)$ had a $33 \%$ higher aOR for any motor vehicle crash, while those sleeping 5 or fewer hours per night $(n=235)$ had a $47 \%$ higher aOR. Those who reported sleeping 9 or more hours per night $(n=235)$ had the lowest crash risk, with an aOR $24 \%$ lower than those sleeping 7 or 8 hours per night. Considering usual sleep duration of 7 to 8 hours per night as normative, the population-attributable fraction of
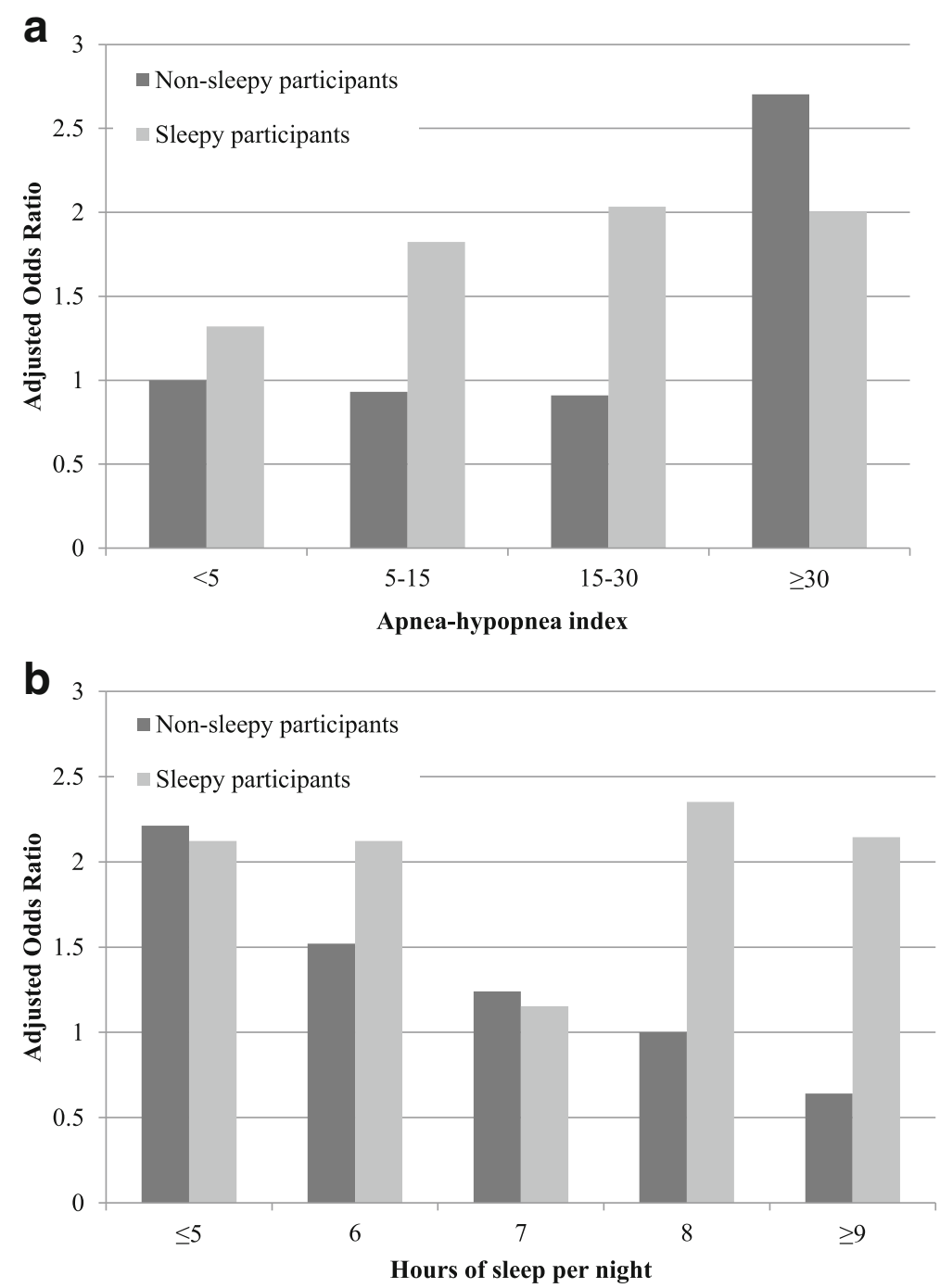

Fig. 2 The relation of sleep apnea (a) and reported usual sleep duration (b) to motor vehicle crash risk, adjusted for age, sex, and miles driven. Data are shown separately for non-sleepy (black, $n=2402$ ) and sleepy (gray, $n=799$ ) participants, where sleepiness is defined as a score $\geq 11$ on the Epworth Sleepiness Scale. The referent group for $\mathbf{a}$ is non-sleepy participants with apnea-hypopnea index $<5$. The referent group for $\mathbf{b}$ is non-sleepy participants reporting a usual sleep duration of 8 hours per night 
motor vehicle crashes related to sleep duration of 6 or fewer hours per night was $9 \%$.

Additional logistic regression models were performed to explore a possible interaction between sleep apnea and sleep duration on risk of motor vehicle crash. While it appears that increased crash risk in those with mild to moderate sleep apnea is present only in those sleeping 6 or fewer hours per night, and that increased crash risk in those with severe sleep apnea is present irrespective of usual sleep duration (Fig. 3), there was no significant interaction between sleep duration and sleep apnea severity with respect to crash risk (interaction term $p=0.65$ ).

The estimated effects of sleep apnea or sleep duration on crash risk were not significantly different between men and women, and were not meaningfully affected by additional adjustment for body mass index, sleeping pill use, smoking history, usual alcohol consumption, or consumption of caffeinated beverages. Effects were also similar between younger and older participants, whether stratified on age 65 or age 70 . Treatment for sleep apnea with continuous positive airway pressure, surgery, or an oral appliance at any time since the baseline sleep study was reported by $2.2 \%$ of the study sample, including $4.7 \%$ of those with AHI $>5$, although treatment adherence and the timing of treatment initiation are not known. However, excluding from the analysis those who reported sleep apnea treatment had no meaningful impact on the association of either sleep apnea or sleep duration with crash risk. A sensitivity analysis that included the 1431 participants who completed the driving questionnaire but were excluded from analysis due to missing data for miles driven per year, adjusted for age and sex but not for miles driven, showed little change in effect estimates or tests of significance.
Self-reported excessive sleepiness was strongly associated with crash risk (Table 2 and Fig. 2). Despite this, adjustment for Epworth Sleepiness Scale score had little impact on the estimated risk associated with sleep apnea (aOR 1.13 per 10-unit increase in AHI) or usual sleep duration (aOR 1.12 per hour shorter sleep duration). Moreover, when the analysis is limited to the $75 \%$ of individuals without self-reported excessive sleepiness, the association of sleep apnea and usual sleep duration with motor vehicle crash risk was not diminished (Table 2). Indeed, the impact of short usual sleep duration on crash risk is most evident in those individuals who do not perceive themselves to be excessively sleepy.

\section{Discussion}

In this community-dwelling cohort of middle-aged and older adults, the presence of sleep deficiency, whether due to short sleep duration or to sleep fragmentation from sleep apnea, was associated with a significantly increased risk of motor vehicle crashes, even among those who did not report excessive sleepiness. While one must be cautious in drawing a causal inference from observational data, the $19 \%$ estimate of motor vehicle crashes attributable to sleep deficiency in this analysis is consistent with estimates from the Virginia Tech $100 \mathrm{car}$ naturalistic driving study that $22 \%$ of actual and nearcrash incidents are attributable to drowsy driving [30], the Institute of Medicine's estimate that $20 \%$ of serious motor vehicle crash injuries are attributable to sleep disorders and sleep deprivation [4], and the recent estimate that $21 \%$ of fatal crash injuries are attributable to drowsy driving [31].

The effect noted in the present study for those with severe sleep apnea is similar to that reported in clinically

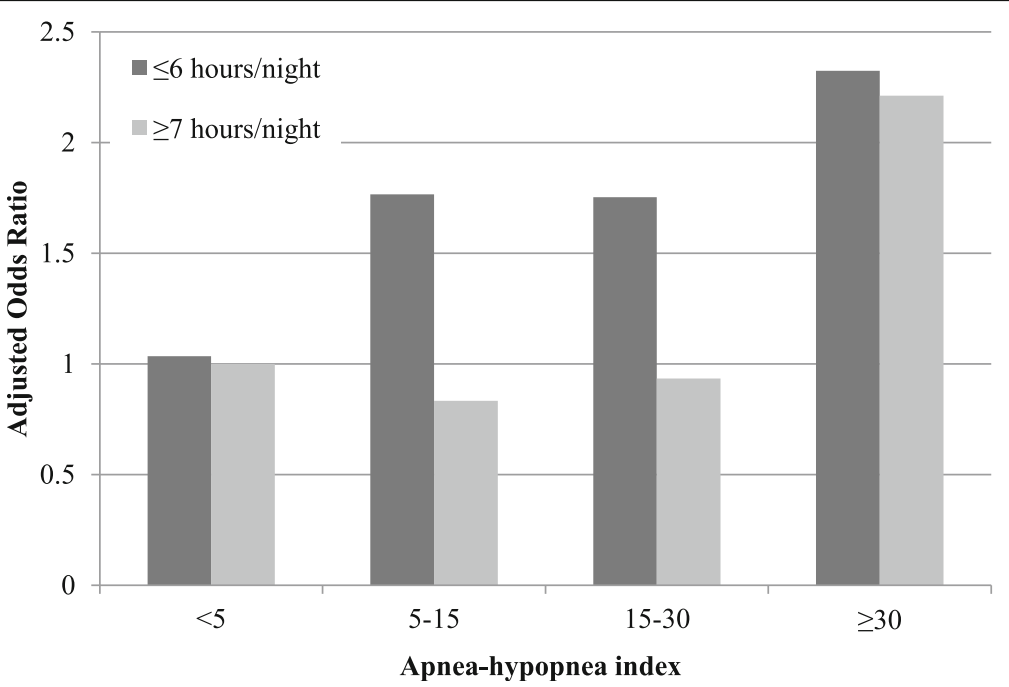

Fig. 3 The joint association of sleep apnea and reported usual sleep duration to motor vehicle crash risk. The referent group is individuals with an apnea-hypopnea index $<5$ and a usual sleep duration of $\geq 7$ hours per night 
diagnosed sleep apnea patients [18, 19], with considerably smaller effects noted in those with milder sleep apnea. Individuals with mild to moderate sleep apnea and self-reported daytime sleepiness more closely resemble clinically diagnosed sleep apnea patients, however, and in this group the elevation in crash risk was also similar to that reported in clinically diagnosed patients. Short sleep duration may also increase crash risk among individuals with mild to moderate sleep apnea (Fig. 3), although further study with a larger sample is needed to verify this suggestive finding. The effects noted in this study are similar to those reported from the Wisconsin Sleep Cohort for risk of any motor vehicle crash over 5 years, although smaller than the risk observed in that cohort for multiple crashes [32]. There were too few participants reporting multiple crashes over the 1-year reporting interval for this study to replicate the Wisconsin analysis. Sleepiness is a widely recognized cause of motor vehicle crashes, a finding also confirmed in the present study. While sleep apnea is an important cause of excessive sleepiness, many individuals with sleep apnea in the general community do not report this symptom [13, 22]. In the present study, severe sleep apnea was associated with increased crash risk even among those without self-reported excessive sleepiness. Together with evidence that excess mortality in sleep apnea is independent of excessive sleepiness [33], these data indicate that treatment is warranted for many apparently asymptomatic patients who deny excessive sleepiness. These data support the conclusion that it is inappropriate to insist that the diagnosis and treatment of obstructive sleep apnea syndrome require excessive daytime sleepiness, which was standard practice for many years [34]. Such a requirement minimizes the prevalence of obstructive sleep apnea and would prevent the nearly half of patients with severe obstructive sleep apnea who do not report excessive sleepiness from obtaining an adequate diagnosis and thorough treatment, thereby perpetuating serious health problems and increased motor vehicle crash risk. These data support recent American Academy of Sleep Medicine diagnostic criteria that do not require the presence of excessive sleepiness for the diagnosis of severe obstructive sleep apnea [20].

In response to accumulating evidence of the potential adverse cardiovascular, metabolic, and performance consequences of insufficient sleep, the National Sleep Foundation, followed by the American Academy of Sleep Medicine and the Sleep Research Society, recently issued evidence-based recommendations that adults should sleep 7 or more hours per night for optimal health [35]. In the present study, habitual sleep durations of less than 7 hours per night are strongly associated with increased motor vehicle crash risk, even among those who do not report excessive sleepiness. A prior casecontrol study in a general population sample found that drivers in crashes involving death or hospitalization were more likely than control drivers to report having slept 5 or fewer hours the previous night, although the impact of habitual sleep duration was not assessed [36]. While reported sleepiness at the time of the crash was strongly associated with crash risk, usual sleepiness as measured by the Epworth Sleepiness Scale was not [36]. In a prospective study of new drivers aged 17-24 years, those who reported habitually sleeping 6 or fewer hours per night had an increased crash risk compared to those sleeping more than 6 hours per night, especially for crashes between the hours of $8 \mathrm{pm}$ and $6 \mathrm{am}$. That study did not evaluate the role of sleepiness [37]. Like the present study, these prior studies suggest increased crash risk in very short sleepers. However, we further found that individuals who habitually sleep 9 or more hours per night have an even lower crash risk than those sleeping the recommended minimum of 7 hours per night, consistent with evidence that individuals who habitually sleep 7 to 8 hours per night carry greater sleep debt than those who habitually sleep 9 hours or more [38]. In commercial drivers, those with an actigraphy-measured sleep duration of 7 to 8 hours per night had a greater sleep propensity and poorer performance on sustained vigilance tasks compared to drivers whose usual sleep duration was greater than 8 hours per night [39]. Thus, the true fraction of motor vehicle crashes attributable to sleep restriction may well exceed 9\%. In the present study, when those sleeping 9 or more hours per night are considered the referent group for crash risk, the estimated population-attributable fraction of crashes due to insufficient sleep rises to $30 \%$. Together with the populationattributable fraction of motor vehicle crashes due to sleep apnea of $10 \%$, this would raise the population-attributable fraction of motor vehicle crashes due to sleep deficiency to $40 \%$, which is considerably higher than prior estimates based largely on self-reporting.

It remains to be determined why many people with severe sleep apnea or short sleep duration in this study did not report excessive sleepiness, even though their elevated crash risk suggests that their driving performance was impaired. Sleep deficiency might impair driving performance by decreasing vigilance even in the absence of increased sleepiness as measured by the Epworth Sleepiness Scale. Alternatively, usual sleepiness may be masked by the use of stimulants such as caffeine or by sympathetic activation secondary to sleep apnea or sleep deprivation. A reduced perception of sleepiness, particularly among those inured to the daytime consequences of chronic sleep deficiency, is also likely, and is consistent with the finding that subjective measures of sleepiness plateau after several days of restricted sleep 
duration, despite continued declines in objective measures of performance such as psychomotor vigilance [10, 11]. The Epworth Sleepiness Scale, although a clinical standard for two decades, may not be a sufficiently sensitive tool to detect a moderate degree of excessive sleepiness. Participants may also be reluctant to admit to excessive sleepiness, a symptom that many regard as pejorative, even in a research setting in which issues of liability are limited. This is likely to be even more problematic in settings in which an acknowledgment of sleepiness may have adverse occupational or legal consequences, e.g., with respect to maintaining a commercial driver's license [40]. Objective tests of sleepiness may be more appropriate in this setting [41].

One potential limitation of this study is the use of selfreport measures of driving and crash history. While motor vehicle crashes documented in police reports are the gold standard for accident reporting, past studies have shown that self-reporting is fairly accurate when reporting on accidents occurring within the prior year, as was done in this study. In a study of adult drivers aged 70 and older, there was substantial agreement between self-report and state-recorded motor vehicle crashes (kappa $=0.64)$ [42], with agreement as high as $85 \%$ between self- and police-reported crashes among younger drivers [43]. Moreover, the rate of motor vehicle crashes reported by the participants in this study (694 per 10,000 person-years or 696 per million miles driven) is quite similar to the rate of motor vehicle crashes reported by the AAA Foundation for Traffic Safety for U.S. adults (650 per 10,000 person-years or 578 per million miles driven) [44]. The strong association between crash risk and miles driven is also internally consistent. An important strength of this study is the prospective assessments of sleep apnea, sleep habits, and sleepiness, which are, therefore, not biased by subsequent crash history. Although these measures were made 1 year prior to the start of the reporting interval for driving history, they are fairly stable over this interval of time $[27,45]$ and variation would in any case bias toward a null result. Another important strength of the study is that it reflects the experience of a large community-dwelling cohort, rather than a cohort of clinically referred patients, and its results are, therefore, more generalizable to the public at large.

\section{Conclusions}

Sleep deficiency resulting from either short sleep duration or sleep apnea is associated with a substantial increase in risk of motor vehicle crashes in the general population. These findings underscore the importance of developing accessible, valid, and objective biomarkers for determining impairment due to sleep deficiency. They further highlight the importance of ensuring that sleep apnea is effectively identified, diagnosed, and appropriately treated. Finally, in conjunction with the adverse metabolic consequences of insufficient sleep, these findings argue for public health efforts to improve adult sleep health.

\section{Abbreviations \\ AHI: Apnea-hypopnea index aORAdjusted odds ratio; MVC: Motor vehicle crash}

\section{Acknowledgments}

No additional acknowledgments outside of listed authors.

\section{Funding}

The Sleep Heart Health Study was supported by National Heart, Lung and Blood Institute cooperative agreements U01HL53940 (University of Washington), U01HL53941 (Boston University), U01HL53938 (University of Arizona), U01 HL53916 (University of California, Davis), U01HL53934 (University of Minnesota), U01HL53931 (New York University), U01HL53937 and U01HL64360 (Johns Hopkins University), U01HL63463 (Case Western Reserve University), and U01HL63429 (Missouri Breaks Research). A list of Sleep Heart Health Study investigators, staff, and their participating institutions is available on the Sleep Heart Health Study website (http://jhuccs1.us/shhs/details/investigators.htm). This work was also supported in part by the following grants: HL095472, HL94654, and U01HL111478 from the National Heart Lung and Blood Institute; P01AG009975 from the National Institute on Aging; GM105018 from the National Institute of General Medical Sciences; NCC9-119, NNX10AF47G, and cooperative agreement NCC9-58 from the National Aeronautics and Space Administration; HFP02801 and HFP02802 from the National Space Biomedical Research Institute; and EMW-2010-FP-00521 from the Federal Emergency Management Agency. Under the cooperative agreement mechanism that is funding the Sleep Heart Health Study, a representative of the National Heart, Lung and Blood Institute participated as a non-voting member in steering committee discussions. The funding agency had no other role in the design of the study and had no role in the collection, analysis, or interpretation of the data or in writing the manuscript.

\section{Availability of data and materials}

The full Sleep Heart Health Study protocol, including the sleep habits, sleepiness, and driving history questionnaires, are available at http:// jhuccs1.us/shhs/details/studydoc.htm. Most of the data analyzed in the current study are available through the National Sleep Research Resource at https://sleepdata.org/, exclusive of individuals not providing consent for inclusion in these public datasets. The full datasets used in this analysis are available from the corresponding author on reasonable request.

\section{Authors' contributions}

DJG was responsible for study design, acquisition, analysis and interpretation of data, and drafting the manuscript. JME and MTB contributed to interpretation of the data and critical revision of the manuscript. CAC contributed to design and interpretation of the analysis and drafting and critical revision of the manuscript. All authors provided approval of the final version of the manuscript and agree to be accountable for all aspects of the work.

\section{Ethics approval and consent to participate}

The protocol was approved by the institutional review board of each participating center and signed informed consent was provided by each participant. The IRB/Human Subjects Committee protocol numbers are as follows: Boston University School of Medicine: H-22384 Johns Hopkins University: H.34.94.05.23.A University of Minnesota: 9406M8378 New York University: H8185 University of Pittsburgh: 9406129 University of California at Davis: 200210023 University of Arizona: 94-0248-01.

\section{Consent for publication}

Not applicable.

\section{Competing interests}

DJG has received consulting fees or served as a paid member of scientific advisory boards for ResMed Corporation, VIVUS, Inc., and T. Leland Seeger \& Associates, Inc.

JME has received consulting fees from Bose Corporation and Invenergy Renewables. 
MTB has received funding from the Center for Integration of Medicine and Innovative Technology, the Milton Family Foundation, the MGH-MIT Grand Challenge, the American Sleep Medicine Foundation, and the Department of Neurology. He has a patent pending on a home sleep-monitoring device, has research agreements with MC10 and Insomnisolv, consulting agreements with McKesson, International Flavors and Fragrances, and Apple, Inc., serves as a medical monitor for Pfizer, and has provided expert testimony in sleep medicine. None of these entities had any role in the study.

CAC has received consulting fees from or served as a paid member of scientific advisory boards for Bose, Boston Red Sox, Columbia River Bar Pilots, Institute of Digital Media and Child Development, Purdue Pharma, Samsung, and Vanda Pharmaceuticals. He owns an equity interest in Vanda Pharmaceuticals. He has served as an expert witness on various legal cases related to sleep and circadian rhythms and he has received research support from Optum, San Francisco Bar Pilots, Schneider, Sysco, Philips Respironics, Vanda Pharmaceuticals, and the State of Washington Board of Pilotage Commissioners. The Harvard Medical School Sleep and Health Education Program and Brigham Sleep Health (CAC) have received funding for educational activities from Cephalon, Jazz Pharma, ResMed, Takeda Pharmaceuticals, Sanofi-Aventis, Sepracor, Simmons, and Mary Ann \& Stanley Snider via Combined Jewish Philanthropies. CAC is the incumbent of an endowed professorship provided to Harvard University by Cephalon, and holds several process patents in the specialty of sleep and circadian rhythms (e.g., photic resetting of the human circadian pacemaker). CAC has received royalties from Houghton Miflin Harcourt, and from Koninklijke Philips Electronics/ Philips Respironics for the Actiwatch-2 and Actiwatch Spectrum devices. CAC's interests were reviewed and are managed by Brigham Health and Partners HealthCare in accordance with their conflict of interest policies.

\section{Publisher's Note}

Springer Nature remains neutral with regard to jurisdictional claims in published maps and institutional affiliations.

\section{Author details}

'Division of Sleep and Circadian Disorders, Departments of Medicine and Neurology, Brigham \& Women's Hospital, 221 Longwood Ave, BLI 225E, Boston, MA 02115, USA. VA Boston Healthcare System, Boston, MA, USA. ${ }^{3}$ Division of Sleep Medicine, Harvard Medical School, Boston, MA, USA. ${ }^{4}$ Department of Neurology, Massachusetts General Hospital, Boston, MA, USA.

\section{Received: 17 November 2017 Accepted: 12 February 2018}

\section{Published online: 20 March 2018}

\section{References}

1. National Center on Sleep Disorders Research. National Institutes of Health Sleep Disorders Research Plan; NIH Publication No. 11-7820; November 2011.

2. Hammond EC. Some preliminary findings on physical complaints from a prospective study of 1,064,004 men and women. Am J Public Health Nations Health. 1964:54:11-23.

3. Kripke DF, Simons RN, Garfinkel L, Hammond EC. Short and long sleep and sleeping pills. Is increased mortality associated? Arch Gen Psychiatry 1979;36:103-16.

4. Colten HR, Altevogt BM, editors. Sleep disorders and sleep deprivation: an unmet public health problem. Washington, D.C.: The National Academies Press; 2006.

5. Connor J, Whitlock G, Norton R, Jackson R. The role of driver sleepiness in car crashes: a systematic review of epidemiological studies. Accid Anal Prev. 2001;33:31-41

6. Stutts JC, Wilkins JW, Scott Osberg J, Vaughn BV. Driver risk factors for sleeprelated crashes. Accid Anal Prev. 2003:35:321-31.

7. Drake C, Roehrs T, Breslau N, Johnson E, Jefferson C, Scofield H, et al. The 10 -year risk of verified motor vehicle crashes in relation to physiologic sleepiness. Sleep. 2010;33:745-52.

8. Centers for Disease Control and Prevention. Drowsy Driving - 19 States and the District of Columbia. MMWR. 2013:61:1033-7.

9. Van Dongen HP, Baynard MD, Maislin G, Dinges DF. Systematic interindividual differences in neurobehavioral impairment from sleep loss: evidence of trait-like differential vulnerability. Sleep. 2004;27:423-33.

10. Van Dongen HP, Maislin G, Mullington JM, Dinges DF. The cumulative cost of additional wakefulness: dose-response effects on neurobehavioral functions and sleep physiology from chronic sleep restriction and total sleep deprivation. Sleep. 2003;26:117-26.
11. Belenky G, Wesensten NJ, Thorne DR, Thomas DL, Sing HC, Redmond DP, et al. Patterns of performance degradation and restoration during sleep restriction and subsequent recovery: a sleep dose-response study. J Sleep Res. 2003:12:1-12.

12. Young T, Palta M, Dempsey J, Skatrud J, Weber S, Badr S. The occurrence of sleepdisordered breathing among middle-aged adults. N Engl J Med. 1993:328:1230-5.

13. Peppard PE, Young T, Barnet JH, Palta M, Hagen EW, Hla KM. Increased prevalence of sleep-disordered breathing in adults. Am J Epidemiol. 2013;177:1006-14.

14. Somers VK, White DP, Amin R, Abraham WT, Costa F, Culebras A, et al. Sleep apnea and cardiovascular disease: an American Heart Association/ American College of Cardiology Foundation scientific statement. Circulation. 2008:118:1080-111.

15. Gottlieb DJ, Yenokyan G, Newman AB, O'Connor GT, Punjabi NM, Quan SF, et al. A prospective study of obstructive sleep apnea and incident coronary heart disease and heart failure: The Sleep Heart Health Study. Circulation. 2010:122:352-60.

16. Redline S, Yenokyan G, Gottlieb DJ, Shahar E, O'Connor GT, Resnick HE, et al. Obstructive sleep apnea and incident stroke: The Sleep Heart Health Study. Am J Respir Crit Care Med. 2010;182:269-77.

17. Marin JM, Agusti A, Villar I, Former M, Nieto D, Carrizo SJ, et al. Association between treated and untreated obstructive sleep apnea and risk of hypertension. JAMA. 2012;307:2169-76.

18. Sassani A, Findley L, Kryger M, Goldlust E, George C, Davidson TM. Reducing motor-vehicle collisions, costs, and fatalities by treating obstructive sleep apnea syndrome. Sleep. 2004;27:453-8.

19. Tregear S, Reston J, Schoelles K, Phillips B. Obstructive sleep apnea and risk of motor vehicle crash: systematic review and meta-analysis. J Clin Sleep Med. 2009:5:573-81.

20. American Academy of Sleep Medicine. International Classification of Sleep Disorders. 3rd ed. Darien IL: American Academy of Sleep Medicine; 2014.

21. US Preventive Services Task Force, Bibbins-Domingo K, Grossman DC, Curry SJ, Davidson KW, Epling JW Jr, et al. Screening for Obstructive Sleep Apnea in Adults: US Preventive Services Task Force Recommendation Statement, vol. 317; JAMA, 2017. p. 407-14.

22. Kapur VK, Baldwin CM, Resnick HE, Gottlieb DJ, Nieto FJ. Sleepiness in patients with moderate to severe sleep-disordered breathing. Sleep. 2005;28:472-7.

23. Quan SF, Howard BV, Iber C, Kiley JP, Nieto FJ, O'Connor GT, et al. The Sleep Heart Health Study: design, rationale, and methods. Sleep. 1997;20:1077-85.

24. Lind BK, Goodwin JL, Hill JG, Ali T, Redline S, Quan SF. Recruitment of healthy adults into a study of overnight sleep monitoring in the home: experience of the Sleep Heart Health Study. Sleep Breath. 2003;7:13-24.

25. Redline S, Sanders MH, Lind BK, Quan SF, Iber C, Gottlieb DJ, et al. Methods for obtaining and analyzing unattended polysomnography data for a multicenter study. Sleep Heart Health Res Group. Sleep. 1998:21:759-67.

26. Whitney CW, Gottlieb DJ, Redline S, Norman RG, Dodge RR, Shahar E, et al. Reliability of scoring respiratory disturbance indices and sleep staging. Sleep. 1998;21:749-57

27. Gottlieb DJ, Punjabi NM, Newman AB, Resnick HE, Redline S, Baldwin CM, et al. Association of sleep time with diabetes mellitus and impaired glucose tolerance. Arch Intern Med. 2005:165:863-7.

28. Johns MW. A new method for measuring daytime sleepiness: The Epworth Sleepiness Scale. Sleep. 1991:14:540-5.

29. Miettinen OS. Proportion of disease caused or prevented by a given exposure, trait or intervention. Am J Epidemiol. 1974:99:325-32.

30. Dingus TA, Klauer SG, Neale VL, Petersen A, Lee SE, Sudweeks J, et al. The 100-Car Naturalistic Driving Study, Phase II-Results of the 100-Car Field Experiment DOT HS 810 593: National Highway Traffic Safety Administration. Washington, D.C.: USDOT; 2006.

31. Tefft BC. Prevalence of motor vehicle crashes involving drowsy drivers, United States, 2009-2013. Washington, D.C: AAA Foundation for Traffic Safety; 2014

32. Young T, Blustein J, Finn L, Palta M. Sleep-disordered breathing and motor vehicle accidents in a population-based sample of employed adults. Sleep. 1997:20:608-13

33. Young T, Finn L, Peppard PE, Szklo-Coxe M, Austin D, Nieto FJ, et al. Sleep disordered breathing and mortality: eighteen-year follow-up of the Wisconsin sleep cohort. Sleep. 2008;31:1071-8.

34. Douglas NJ. Chapter 265. Sleep Apnea. In: Longo DL, Fauci AS, Kasper DL, Hause $\mathrm{SL}$, Jameson J, Loscalzo J. Harrison's Principles of Internal Medicine, 18e New York NY: McGraw-Hill; 2012. http://accessmedicine.mhmedical.com.ezp-prod1.hul. harvard.edu/Content.aspx?bookid=331 Sionid=40727048. Accessed 17 Feb 2018. 
35. Watson NF, Badr MS, Belenky G, Bliwise DL, Buxton OM, Buysse D, et al. Recommended amount of sleep for a healthy adult: a joint consensus statement of the American Academy of Sleep Medicine and Sleep Research Society. Sleep. 2015;38:843-4.

36. Connor J, Norton R, Ameratunga S, Robinson E, Civil I, Dunn R, et al. Driver sleepiness and the risk of serious injury to car occupants: population based case control study. BMJ. 2002;324:1125.

37. Martiniuk ALC, Senserrick T, Lo S, Williamson A, Du W, Grunstein RR, et al. Sleep-deprived young drivers and the risk for crash: The DRIVE Prospective Cohort Study. JAMA Pediatr. 2013;167:647-55.

38. Klerman EB, Dijk DJ. Interindividual variation in sleep duration and its association with sleep debt in young adults. Sleep. 2005;28:1253-9.

39. Pack Al, Maislin G, Staley B, Pack FM, Rogers WC, George CF, et al. Impaired performance in commercial drivers: role of sleep apnea and short sleep duration. Am J Respir Crit Care Med. 2006;174:446-54.

40. Sharwood LN, Elkington J, Stevenson M, Grunstein RR, Meuleners L, Ivers $\mathrm{RQ}$, et al. Assessing sleepiness and sleep disorders in Australian longdistance commercial vehicle drivers: self-report versus an "at home" monitoring device. Sleep. 2012;35:469-75.

41. Philip P, Sagaspe P, Taillard J, Chaumet G, Bayon V, Coste O, et al. Maintenance of wakefulness test, obstructive sleep apnea syndrome, and driving risk. Ann Neurol. 2008;64:410-6.

42. Singletary BA, Do AN, Donnelly JP, Huisingh C, Mefford MT, Modi R, et al. Self-reported vs state-recorded motor vehicle collisions among older community dwelling individuals. Accid Anal Prev. 2017;101:22-7.

43. Boufous $S$, Ivers R, Senserrick T, Stevenson M, Norton R, Williamson A. Accuracy of self-report of on-road crashes and traffic offences in a cohort of young drivers: the DRIVE study. Inj Prev. 2010;16:275-7.

44. Tefft BC. Motor vehicle crashes, injuries, and deaths in relation to driver age: United States, 1995-2010. Washington, D.C: AAA Foundation for Traffic Safety; 2012.

45. Newman AB, Foster G, Givelber R, Nieto FJ, Redline S, Young T. Progression and regression of sleep-disordered breathing with changes in weight: the Sleep Heart Health Study. Arch Intern Med. 2005;165:2408-13.

\section{Submit your next manuscript to BioMed Central and we will help you at every step:}

- We accept pre-submission inquiries

- Our selector tool helps you to find the most relevant journal

- We provide round the clock customer support

- Convenient online submission

- Thorough peer review

- Inclusion in PubMed and all major indexing services

- Maximum visibility for your research

Submit your manuscript at www.biomedcentral.com/submit 